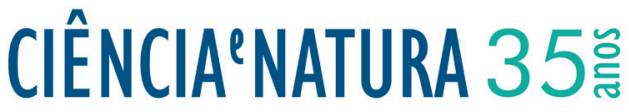

\section{Incertezas das projeções de mudanças climáticas: Análise preliminar}

\author{
Uncertainties of Climate Change Projections: Preliminary analysis
}
Thalyta Soares dos Santos ${ }^{1}$, David Mendes ${ }^{2}$, Aline Anderson de Castro ${ }^{3}$ e Allan Rodrigues Silva ${ }^{4}$

${ }^{1}$ Pós-Graduação em Ciências Climáticas, UFRN

${ }^{2}$ Escola de Ciência e Tecnologia, UFRN

${ }^{3}$ Instituto Nacional de Pesquisas Espaciais, INPE

${ }^{4}$ Pós-Graduação em Ciências Climáticas, UFRN

\begin{abstract}
Resumo
Embora o enorme avanço científico e computacional das últimas décadas tenha possibilitado um maior e melhor entendimento do sistema climático, e assim contribuído para as análises das possíveis causas e futuros impactos das mudanças climáticas globais, as incertezas que permeiam as projeções de clima, tais como as reportadas pelo IPCC AR4, ainda continuam muito grandes, principalmente em escala regional. Este trabalho avalia as incertezas envolvidas nas projeções de precipitação na América do Sul no século XXI. Simulações de clima X modelos de circulação geral da atmosfera disponível no Coupled Model Intercomparison Project 5th Phase (CMIP5) são avaliadas de acordo com o método Reliability Ensemble Averaging (REA). Os resultados deste estudo mostraram a confiabilidade do conjunto de modelos foi satisfatória para toda a América do Sul no cenário RCP2.6, indicando boa confiabilidade dos modelos nessa região.
\end{abstract}

Palavras-chave: Reliability Ensemble Averaging. América do Sul. Confiabilidade

\begin{abstract}
Although the huge scientific and computational advances of recent decades has enabled a greater understanding of the climate system and contributed to the analysis of possible causes and future impacts of global climate change, the uncertainties that pervade the climate projections, such as those reported by IPCC AR4, are still very large, particularly on a regional scale. This study evaluates the uncertainties involved in projections in South America rainfall in the the 20th and 21st century. Simulations of three climate general circulation models of the atmosphere Coupled Model Intercomparison Project 5th Phase Project (CMIP5) are evaluated according to the Reliability Ensemble Averaging (REA) method. The results of this study showed the ensemble reliability has been satisfactory for all South America in the RCP2.6 scenario, indicating good reliability of the models in this region.
\end{abstract}

Keywords: Reliability Ensemble Averaging. South America. Reliability 


\section{Introdução}

Os modelos climáticos são baseados em princípios físicos bem estabelecidos e são utilizados para reproduzir observações características do clima recente e alterações do clima passado. O grande problema é na confiabilidade que os Modelos de Circulação Geral Atmosfera-Oceano (MCGOAs) apresentam em níveis regionais. A confiabilidade nessas estimativas é maior para algumas variáveis (ex. temperatura) que para outras (ex. precipitação).

Giorgi e Mearns (2002) afirmam que um dos principais fatores de incerteza das simulações, é que diferentes MCGOAs podem simular diferentes mudanças regionais, mesmo sob o mesmo cenário de forçante antropogênica (por exemplo, KITTEL et al., 1998; GIORGI; FRANCISCO, 2000; WHETTON et al., 1996) e é muito difícil saber qual dos modelos climáticos globais (MCG) é mais responsável por essas diferenças. Giorgi e Mearns (2002) apontam que uma avaliação abrangente das projeções de mudanças regionais precisa ser baseada na informação coletiva de um conjunto de simulações de modelos.

Quantificar as incertezas nas projecções de cenários climáticos futuros utilizados para as avaliações de impacto tem sido identificado como uma necessidade de estudos e inspirou diversos trabalhos (por exemplo, JONES, 2000a, b; NEW; HULME, 2000; KATZ, 2001).

Diversas formas têm sido utilizadas para tentar obter as incertezas. Uma delas apontada por Valverde e Marengo (2008) é obter um panorama único para um determinado cenário é através de uma média dos diferentes modelos (ensemble), de tal forma, a visualizar apenas as áreas que são comuns e representativas a todos os modelos. Pois, as áreas que não são comuns terminamse anulando ou minimizando, restando apenas as mais significativas. Vários trabalhos como Tebaldi et al. (2006) têm adotado a técnica de ensemble, a fim de minimizar e quantificar as incertezas através de índices estatísticos (KUMAR et al., 2001), e facilitar a análise do que pode ser um comportamento futuro(TAYLOR et al., 2005).

Um método bastante usado para sintetizar os resultados de uma previsão por conjunto é a produção de uma média simples, onde para cada membro é atribuído igual probabilidade de ocorrência. Esta metodologia tem-se demonstrado hábil em produzir resultados mais próximos aos observados do que qualquer membro isolado do conjunto (EBERT, 2001). Outra técnica para lidar com as diferentes projeções climáticas dos modelos numéricos é a utilização de aproximações probabilísticas, na qual os resultados de diferentes modelos ou integrações de um mesmo modelo são utilizados para a produção de uma Função de Densidade Probabilistica (FDP, na sigla em inglês) (GIORGI, 2005; COLLINS, 2007). A largura ou espalhamento da FDP representa uma medida da incerteza na projeção, e a integral entre dois limiares estabelecidos indicam a probabilidade de sua ocorrência.

Giogi e Mearns (2002) propuseram a aplicação de um método baseado na média ponderada de diferentes
MCGOAs, levando em consideração a "confiabilidade" de cada modelo, denominado Reliability Ensemble Averaging (REA). No método REA esta confiabilidade leva em consideração a destreza de cada modelo em simular o clima observado (viés) e o grau de convergência de sua projeção para o futuro com relação aos outros modelos do conjunto. Este método também possibilita uma avaliação da credibilidade da média ponderada das projeções, o cálculo de um intervalo de incerteza e a produção de resultados probabilísticos.

De acordo com os problemas expostos acima e a necessidade de melhorar as informações a respeito das simulações climáticas, este trabalho realiza uma análise das incertezas das simulações climáticas nas projeções de mudanças climáticas sobre a América do Sul utilizando o método Reliability Ensemble Averaging.

\section{Materiais e Métodos}

\section{1 Área de Estudo}

Este estudo será aplicado na América do Sul, devido a sua grande extensão territorial são observados diferentes regimes de precipitação e uma grande variabilidade de climas com diferentes características regionais.

Na América do Sul foi utilizada a média dos parâmetros REA em determinadas áreas. Quatro sub-regiões principais na Amazônia: Noroeste e Nordeste, Sudeste e Sudoeste, (NOAMZ, NEAMZ, SEAMZ e SOAMZ, respectivamente), Nordeste Brasileiro (NEB), Sudeste (SE) e a região da Bacia do Prata (LPB), como mostra a figura 1. Estas regiões foram escolhidas segundo o regime de precipitação de cada região.

\subsection{Dados}

Serão utilizadas simulações climáticas de precipitação geradas por três MCGOAs utilizados no CMIP5 (Coupled Model Intercomparison Project 5th Phase): MIROC5, MPI-ESM-LR e CSIRO-Mk3.6.0, em dois cenários de diferentes.

Analisou-se o período de controle, histórico (1980 a 2000) e no cenário RCP2.6, que representa o nível mais baixo da evolução do $\mathrm{CO}_{2}$ (projeção para 2080-2100). Os modelos do CMIP5 serão avaliados com os dados de reanálise do ERA-INTERIM. A resolução espacial dos MCG utilizados variam de aproximadamente $1^{\circ}$ a $2^{\circ}$, para fins de comparação os modelos e a reanálise foram interpolados para uma grade de $2^{\circ} \times 2^{\circ}$.

\subsection{Reability Ensemble Averaging}

No método REA, a variação média, $\tilde{\Delta} \xi$, é dada pela média ponderada dos membros do conjunto. Ou seja, a média do conjunto leva em conta explicitamente o critério de confiabilidade e não pesa igualmente todas 


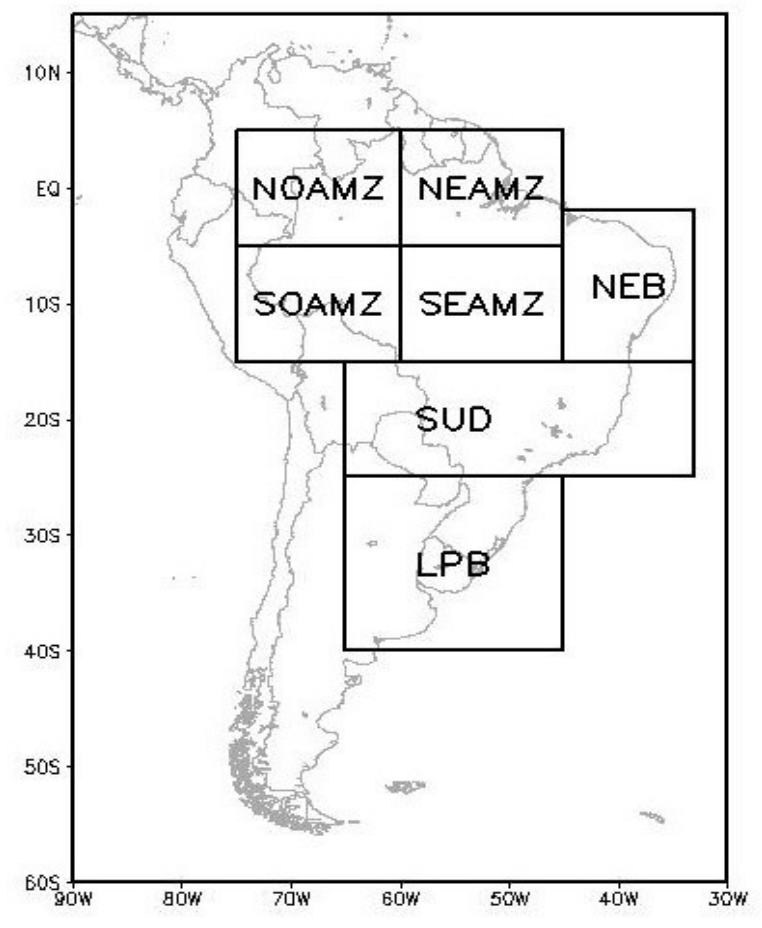

Figura 1 - Região de estudo. As caixas indicam a sub-regiões usadas na análise: Noroeste Nordeste, Sudeste e Sudoeste da Amazônia (NOAMZ, NEAMZ, SEAMZ, SOAMZ), Nordeste do Brasil (NEB), Sudeste do Brasil (SUD) e Bacia da Prata (LPB).

as simulações do modelo.

$$
\bar{\Delta} \xi=\frac{\sum_{i} R_{i} \Delta \xi_{i}}{\sum_{i} R_{i}}
$$

No $R_{i}$ qual é o fator de confiabilidade do modelo definido como:

$$
\begin{gathered}
R_{i}=\left[\left(R_{B, i}\right)^{m} X\left(R_{D, i}\right)^{n}\right]^{\left[\frac{1}{m x n}\right]} \\
R_{i}=\left\{\left[\frac{\epsilon_{\xi}}{\operatorname{abs}\left(B_{\xi, i}\right)}\right]^{m}\left[\frac{\epsilon_{\xi}}{\operatorname{abs}\left(D_{\xi, i}\right)}\right]^{n}\right\}^{[1 /(m x n)]}
\end{gathered}
$$

$R_{B, i}$ é uma paramêtro que mensura o critério de desempenho do modelo enquanto ${ }^{R_{D, i}}$ é uma medida do critério de convergência do modelo. Os parâmetros m e n podem ser utilizados para balancear cada critério. Para os cálculos neste trabalho, $\mathrm{m}$ e $\mathrm{n}$ são assumidos como sendo igual a 1, o que dá igual peso a ambos os critérios. Quando o viés e a distância crescem, a confiabilidade de uma simulação de determinado modelo diminui.

Além disso, RB e RD são definidos como 1 quando $B_{\xi, i}$ e $D_{\xi, i}$ são menores do $\epsilon_{\xi}$ que, respectivamente. Como proposto por Giorgi e Mearns (2002), o paramêtro é a međida da variabilidade natural em 30 anos da variável. A variabilidade natural ( ) é compatada a partir da série temporal das observações regionais com médias das variáveis para o século $\mathrm{XX}$ sobre a área de estudo. Em seguida, é calculada a média móvel de 20 anos da série após remover linearmente a tendência dos dados e estima-se como điferença entre os valores máximo e mínimo.

Uma medida da confiabilidade coletiva dos modelos

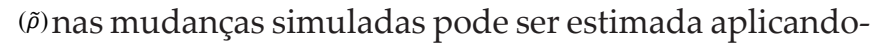
se o operador REA ao fator de confiabilidade, ou seja,

$$
\tilde{\rho}=\tilde{A}(R)=\frac{\sum_{i} R_{i}^{2}}{\sum_{i} R_{i}}
$$

\section{Resultados}

A média REA de precipitação, método que leva em conta confiabilidade de cada modelo (medida pelo desempenho e critérios de convergência dos modelos) para o período de controle e o cenário futuro RCP 2.6 pode ser observado na Figura 2 (a e b, respectivamente). É possível observar que a média da precipitação é bem homogênea no cenário futuro e varia entre 2,5 a $3 \mathrm{~mm} /$ dia 


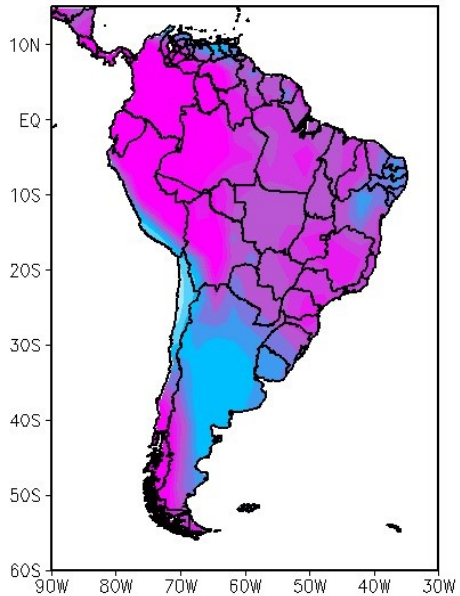

a)

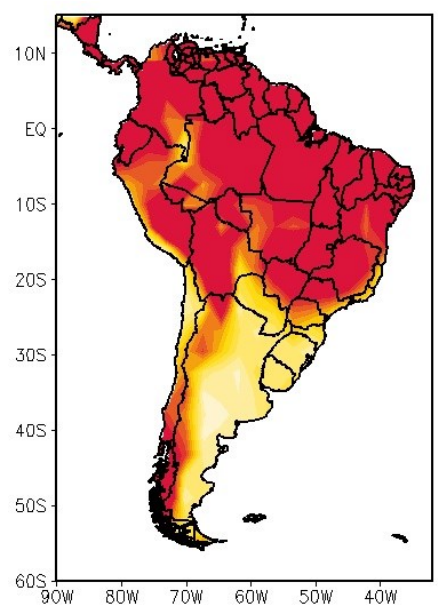

c)

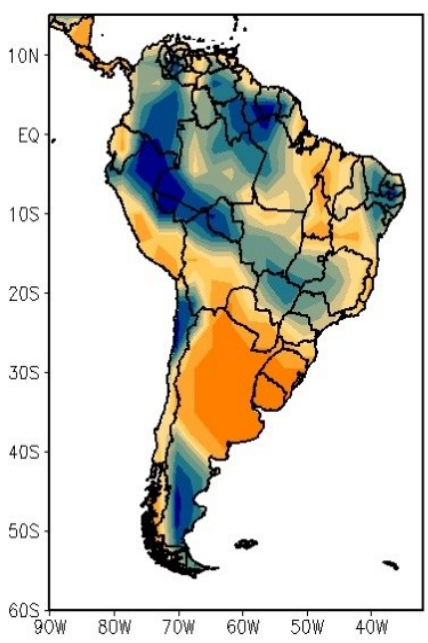

e)
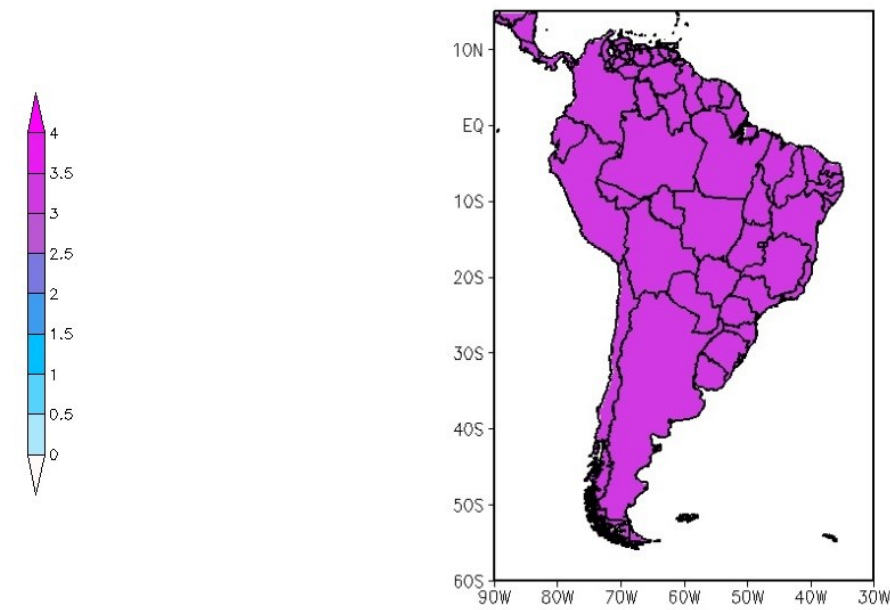

b)

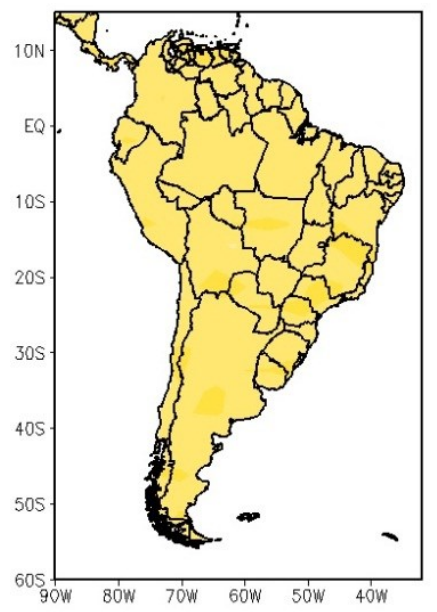

d)

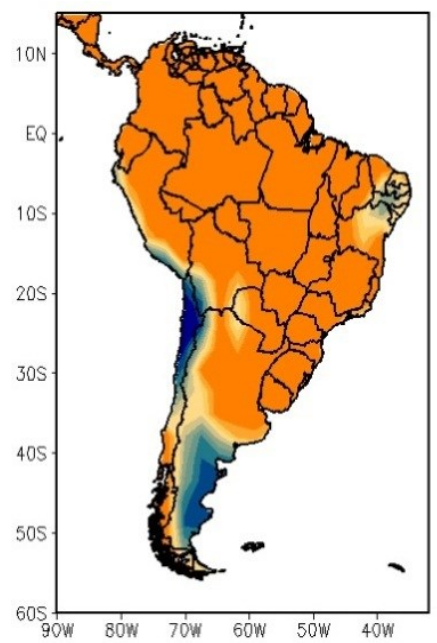

f)

Figura 2 - Média REA (A-B), intervalo de incerteza (C-D) e confiabilidade do conjunto de modelos (E-F) para a precipitação ( $\mathrm{mm} /$ dia), no período de controle (coluna esquerda) e cenário RCP2.6 (coluna direita). 
Tabela 1 - Média do conjunto de modelos $(\overline{\Delta P})$, Média $\operatorname{REA}\left(\Delta P_{R E A}\right)$, intervalo de incerteza ( $\left.\tilde{\delta}_{\Delta P}\right)$, confiabilidade do conjunto $(\rho)$ da precipitação para o verão e o inverno astral no período de 1980-2000

\begin{tabular}{ccccc}
\hline Região & Período & $\overline{\Delta \boldsymbol{P}}$ & $\boldsymbol{\Delta P}_{\boldsymbol{R E A} \boldsymbol{A}}$ & $\boldsymbol{\rho}$ \\
\hline NOAMZ & DJF & 7,16 & 6,7 & 0,88 \\
& JJA & 3,37 & 3,35 & 0,99 \\
NEAMZ & DJF & 4,69 & 4,21 & 0,91 \\
& JJA & 1,80 & 1,55 & 0,97 \\
SEAMZ & DJF & 8,17 & 7,98 & 0,94 \\
& JJA & 0,89 & 0,85 & 0,98 \\
SOAMZ & DJF & 7,17 & 7,16 & 0,99 \\
& JJA & 0,24 & 0,23 & 0,97 \\
NEB & DJF & 6,55 & 5,59 & 0,58 \\
\multirow{2}{*}{ SE } & JJA & 1,04 & 0,76 & 0,79 \\
& DJF & 5,18 & 5,10 & 0,95 \\
LPB & JJA & 0,71 & 0,66 & 0,95 \\
& DJF & 3,46 & 3,39 & 0,74 \\
& JJA & 2,58 & 2,53 & 0,95 \\
\hline
\end{tabular}

em toda América do Sul. Enquanto no cenário histórico a média da precipitação é maior sobre a Região Amazônica (região difícil de simular devido à dificuldade de se obter dados observados) e menor no sul da América do Sul e sobre os Andes.

Nota-se ainda que no período de controle (Figura 2-c) é observado uma grande faixa de incerteza sobre o norte da América do Sul e na região dos Andes, o que representa uma grande discrepância dos modelos nessa região, o que não é observado no cenário RCP 2.6 (Figura 2-d), que apresenta baixa incerteza de acordo com o REA.

Ao verificar a confiabilidade coletiva do conjunto de modelos é possível notar que o cenário futuro apresenta uma grande confiabilidade, exceto na região dos Andes e sul da América do Sul quando comparado a período de controle, que possui baixa confiabilidade ainda sobre o nordeste do Brasil e na região Amazônica. Seth et al. (2010) afirmam que a má representação das condições climáticas atuais sobre Cordilheira dos Andes pode ser relacionada com a grande resolução do modelo que não permite uma boa representação. Stern e Miyakoda, 1995 apontam ainda uma deficiência dos modelos na representação espectral da topografia complexa desta região.

A Tabela 1 mostra os resultados do REA para a precipitação nas principais regiões indicadas na Figura 1 para o período 1980-2000, para o verão e inverno austral. Nas diferenças entre a média simples do modelo e a média REA nota-se que em alguns casos, as estimativas de mudança com os dois métodos são muito próximas umas das outras. Apesar disso, na maioria dos casos a abordagem REA, em grande parte das regiões (exceto em NOAMZ e LPB) reduz a magnitude da variação quando comparado com a média simples do conjunto, o que já esperado já que a variação REA leva em conta a confiabilidade dos modelos. É notável ainda que em DJF a variação é maior que nos meses JJA, o que pode ser devido à maior variabilidade da precipitação neste período.

Na região SEAMZ, onde se localiza a Bacia do Xingú, apresentou as maiores divergências entre os modelos na estação chuvosa. Lucas et al. (2006) ao estudarem a regionalização e distribuição espacial e temporal da precipitação nesta Bacia verificaram que nas partes norte e sudoeste ocorrem as maiores magnitudes de chuvas, principalmente causadas pela atuação dos sistemas atmosféricos conhecidos como Zona de Convergência Intertropical (ZCIT) e Zona de Convergência do Atlântico Sul (ZCAS). Além disso, a região também sofre influência do ENSO (El Niño Oscilação Sul), fenômeno atmosférico -oceânico caracterizado por um aquecimento (El Niño)/ resfriamento(Lã Niña) anormal das águas superficiais no oceano Pacífico Tropical, que acarreta uma diminuição/ aumento nos totais pluviométricos na Amazônia.

Segundo Giorgi e Mearns (2002) os valores de confiabilidade da precipitação devem ser avaliados em relação à magnitude das mudanças. Segundo estes autores, é possível que uma alta confiabilidade indique simplesmente que é a alteração é a variabilidade natural estimada, isto é, que existe elevada confiabilidade numa simulação sem grandes mudanças significativas. Afirma ainda que, os valores superiores a 0,6 são pequenos e dentro da variabilidade estimada. Na Tabela 1 nota-se que para todas as regiões determinadas, exceto em JJA no NEB, a confiabilidade foi superior a 0,7 , indicando uma alta confiabilidade dos dados neste estudo preliminar.

\section{Conclusões}

O objetivo do método REA é minimizar a contribuição de simulações que tem uma desempenho mais pobre na representação do clima. Portanto, pode-se extrair apenas as informações mais confiáveis de cada modelo.

No período de controle quando se leva em conta o 
desempenho de cada modelo em representar o clima atual e a convergência de sua projeção para o conjunto significa na média de conjunto, tal como proposto pelo método REA, nota-se que a variação REA é ligeiramente menor ao longo de todo o continente, quando comparado ao cenário futuro RCP2.6. Porém, mesmo com a média REA mais baixa o período de controle apresentou um intervalo de incerteza claramente maior do que para o as projeções futuras.

A baixa confiabilidade coletiva do conjunto de modelos para o período de controle corrobora com a grande faixa de incerteza observada. Essa confiabilidade aumenta quando faz-se o estudo sazonalmente. A confiabilidade foi acima de 0,8 para quase toda a América do Sul, o que pode indicar que existe uma alta confiabilidade das simulações, sem nenhuma variação significativa. Isto indica que o cenário RCP2.6 quando analisado com um conjunto de modelos, fornece informações bastantes consistentes do clima futuro.

\section{Referências}

COLLINS, M. Ensembles and probabilities: a new era in the prediction of climate change. Phil. Trans. R. Soc. A., 365 (1857), 1957-1970, 2007.

EBERT, E. E. Ability of a poor man's ensemble to predict the probability and distribution of precipitation. Mon. Wea. Rev., 129, 2461-2480, 2001.

GIORGI, F.; FRANCISCO, R. Evaluating uncertainties in the prediction of regional climate change. Geophys. Res. Lett., 27, 1295-1298, 2000.

GIORGI, F.; MEARNS, L. O. Calculation of Average, Uncertainty Range, and Reliability of Regional Climate Changes from AOGCM Simulations via the "Reliability Ensemble Averaging" ( REA ) Method. Journal of Climate, 15, 1141-1158, 2002.

GIORGI, F. Climate change prediction. Clim. Change, 73, 239-265, 2005.

JONES, R. N. Managing uncertainty in climate change projections. Issues for impact assessment. Climate Change, 45, 403-419, 2000a.

JONES, R. N. Analysing the risk of climate change using an irrigation demand model. Climate Res., 14, 89-100, $2000 \mathrm{~b}$.

KATZ, R. W. Techniques for estimating uncertainty in climate change scenarios and impact studies. Climate Res., in press, 2001.
KITTEL, T. G. F., GIORGI, F.; MEEHL, G. A. Intercomparison of regional biases and doubled $\mathrm{CO} 2$ sensitivity of coupled atmosphere-ocean general circulation model experiments. Climate Dyn., 14, 1-15, 1998.

KUMAR, A.; BARNSTON, A.; HOERLING, M. Seasonal Predictions, probabilistic verifications, and ensemble size. Journal Climate 14:1671-1676, 2001.

LUCAS, E. W. M.; SOUSA, F. A. S.; MENEZES, R. H. N.; COSTA, R. F.; OLIVEIRA, L. L.; BARRETO, N. J. C. Variação espacial e temporal da precipitação na bacia hidrográfica do Xingu, Pará. In: CONGRESSO BRASILEIRO DE METEOROLOGIA, XIII, 2006, Florianópolis/SC: Sociedade Brasileira de Meteorologia, 2006.

NEW, M.; HULME, M. Representing uncertainty in climate change scenarios: A Monte-Carlo approach. Integr. Assess., 1, 203-214, 2000:

SETH, A.; ROJAS, M.; RAUSCHER, S. A. CMIP3 projected changes in the annual cycle of the South American monsoon. Clim Change 98:331-357, 2010. doi:10.1007/ s10584-009-9736-6.

STERN, W., MIKAYODA, K. Feasibility of seasonal forecast inferred from multiple GCM simulation. Journal of Climate, v. 8, p. 1071-1085, 1995.

TAYLOR, K. E.; GLECKER, P. J.; E DOUTRIAX. Tracking changes in the performance of AMIP models, in the second Phase of the Atmospheric Model Interconparison Project (AMIP2): Toward Innovative Model Diagnostic, Edited by P.J. Gleckler, WCRP report, in preparation. 2005.

TEBALDI, C.; HAYHOE, K.; ARBLASTER, J.; MEEHL, G. Going to the extremes An intercomparison of modelsimulated historical and future changes in extreme events. No prelo, Climatic Change, 2006.

VALVERDE, M.; MARENGO, J. Cenário future de circulação atmosférica para a climatologia 2011-2040, derivado de uma média multi-model das simulações dos modelos do IPCC AR4. IN: CONGRESSO BRASILEIRO DE METEOROLOGIA, 15, São Paulo. Anais... 2008. CD-ROM.

WHETTON, P. H.; ENGLAND, M. H.; O'FARRELL, S. P.; WATTERSON, I. G.; PITTOCK, A. B. Global comparison of the regional rainfall results of enhanced greenhouse coupled and mixed layer ocean experiments: Implications for climate change scenario devel- opment. Climatic Change, 33, 497-519, 1996. 